\title{
CARDINAL FUNCTIONS ON PIXLEY-ROY HYPERSPACES
}

\author{
SHOZO SAKAI
}

\begin{abstract}
Some cardinal functions on the Pixley-Roy hyperspace $\widetilde{Y}[X]$ of a space $X$ are determined by those on $X$, and conditions of $X$ by cardinal functions from which $\bar{y}[X]$ has or has not some properties, e.g. cosmic, paracompact, ccc, etc. are given.
\end{abstract}

All spaces considered in this paper will be assumed to be infinite $T_{1}$-spaces. Cardinals are the initial ordinals, $\tau$ will always denote an infinite cardinal and $\omega$ is the smallest infinite cardinal. The cardinality of a set $X$ is denoted by $|X|$ and $\mathrm{c}$ is the cardinality of the continuum. The successor cardinal of $\tau$ is denoted by $\tau^{+}$.

The Pixley-Roy hyperspace $\mathcal{F}[X]$ of a space $X$ has as its underlying set the collection of all finite nonempty subsets of $X$. If $A \in \mathscr{F}[X]$, then the basic nbds (= neighbourhoods) of $A$ are $[A, U]=\{S \in \mathscr{F}[X]: A \subset S \subset U\}$, where $U$ is an open set of $X$ containing $A$.

We refer to $[\mathbf{J}]$ for the following cardinal functions-w (weight), $\pi$ ( $\pi$-weight), $n w$ (net weight), d (density), c (cellularity), L (Lindelöf-degree $), p(=e$ (extent) in $[\mathbf{E}]), \chi$ (character), $\psi$ ( pseudo character), $\Psi\left(\Psi(X)=\omega\right.$ iff $X$ is perfect), $\psi_{\Delta}$ (diagonal degree $), \pi \chi(\pi$-character $), t$ (tightness) and psw (point separating weight). However, following Engelking [E], the hereditary density and the hereditary Lindelöf-degree are denoted by $h d$ and $h L$, respectively.

In the following, Theorem 2 is the main result of this paper. Some results in it were given by van Douwen [vD], Lutzer [L] and Przymusiński [P]. Though Proposition 1 is an auxiliary one for Theorem 2, it is interesting in itself.

Proposition 1. If $A \in \mathcal{F}[X]$, then the following hold:

(1) $\psi(A, \mathscr{F}[X])=\max \{\psi(x, X): x \in A\}$.

(2) $\pi \chi(A, \mathscr{F}[X])=\chi(A, \mathscr{F}[X]) \leqslant \max \{\chi(x, X): x \in A\}$.

If $x \in X$, then the following hold:

(3) $\chi(\{x\}, \mathscr{F}[X])=\chi(x, X)$.

(4) $t(x, X) \leqslant t(\{x\}, \widetilde{F}[X])$.

If $X$ is Hausdorff and $A \in \mathscr{F}[X]$, then the following holds:

(5) $\chi(A, \mathscr{F}[X])=\max \{\chi(x, X): x \in A\}$.

Received by the editors November 5, 1982 and, in revised form, February 16, 1983.

1980 Mathematics Subject Classification. Primary 54A25, 54B20; Secondary 54D18, 54D20. 
Proof. (1) Let $A=\left\{x_{1}, \ldots, x_{n}\right\} \in \mathscr{F}[X]$. For each $i \in\{1, \ldots, n\}$, there is a local $\psi$-base $\mathcal{Q}_{i}$ of $x_{i}$ in $X$ such that $\left|\mathcal{Q}_{i}\right| \leqslant \psi\left(x_{i}, X\right)$. Let

$$
\text { Q }=\left\{\cup\left\{U_{i}: i=1, \ldots, n\right\}: U_{i} \in \mathcal{Q}_{i}\right\} \quad \text { and } \mathscr{Q}=\left\{[A, U]: U \in \mathcal{Q l}_{\mathrm{L}}\right\} \text {. }
$$

Then $\mathscr{G}$ is a local $\psi$-base of $A$ in $\mathscr{F}[X]$ and $|\mathscr{T}| \leqslant \max \left\{\psi\left(x_{i}, X\right): i=1, \ldots, n\right\}$. Thus, we have $\psi(A, \mathscr{F}[X]) \leqslant \max \{\psi(x, X): x \in A\}$. Conversely, let $\mathscr{B}$ be a local $\psi$-base of $A$ in $\widetilde{\mathcal{F}}[X]$ and $x \in A$. Then $\mathscr{U}=\{B \backslash(A \backslash\{x\}):[A, B] \in \mathscr{B}\}$ is a local $\psi$-base of $x$ in $X$. Therefore, we have $\psi(x, X) \leqslant \psi(A, \mathscr{F}[X])$ and $\max \{\psi(x, X): x \in A\} \leqslant$ $\psi(A, \mathscr{F}[X])$.

(2) Let $\mathscr{B}$ be a local $\pi$-base of $A$ in $\mathscr{F}[X]$. For each $B \in \mathscr{B}$, we can take an element $A_{B} \in B$ and an open nbd $U_{B}$ of $A_{B}$ in $X$ such that $\left[A_{B}, U_{B}\right] \subset B$. Then, $\mathcal{Q}=\left\{\left[A, U_{B}\right]\right.$ : $\left.B \in \mathscr{G}, A \subset U_{B}\right\}$ is a nbd base of $A$ in $\mathscr{F}[X]$. For, let $V$ be an open nbd of $A$ in $X$. Then, there is $B \in \mathscr{B}$ such that $B \subset[A, V]$. Therefore, $\left[A_{B}, U_{B}\right] \subset B \subset[A, V]$, i.e. $A \subset A_{B} \subset U_{B} \subset V$. Thus, $\left[A, U_{B}\right] \subset[A, V]$ and $\mathscr{Q}$ is a nbd base of $A$ in $\mathscr{F}[X]$. Since $|\mathcal{Q}| \leqslant|\mathscr{H}|$, we have $\chi(A, \mathscr{F}[X]) \leqslant \pi \chi(A, \mathscr{F}[X]) . \pi \chi(A, \mathscr{F}[X]) \leqslant \chi(A, \mathscr{F}[X])$ holds always and the other part is proved similar to (1).

(3) The proof is clear.

(4) Let $H \subset X, x \in X \backslash H$ and $x \in \operatorname{cl}_{X} H$. Let us put $\mathscr{H}=\mathscr{F}[H \cup\{x\}] \backslash\{\{x\}\}$. Then $\{x\} \in \operatorname{cl}_{\bar{G}[X]} \mathcal{H}$. Therefore, there is a subset $\mathscr{N}$ of $\mathscr{H}$ such that $|\mathfrak{N}| \leqslant$ $t(\{x\}, \mathscr{F}[X])$ and $\{x\} \in \operatorname{cl}_{\mathscr{\mathscr { F }}[X]} \mathfrak{T}$. Let $M=(\cup \mathfrak{N}) \backslash\{x\}$. Then $x \in \mathrm{cl}_{X} M$. For, let $V$ be a nbd of $x$ in $X$. Since $\{x\} \in \operatorname{cl}_{\mathscr{F}[X]} \mathscr{N}$, there is an element $A \in[\{x\}, V] \cap \mathscr{N}$. Then, $x \in A \subset V$ and $A \in \mathfrak{N}$. Since $A \neq\{x\}$, we have $V \cap M \neq \varnothing$ and $x \in \operatorname{cl}_{X} M$. Since $M \subset H$ and $|M| \leqslant|\mathscr{T}| \leqslant t(\{x\}, \mathscr{F}[X])$, we have $t(x, X) \leqslant t(\{x\}, \mathscr{F}[X])$.

(5) Let us assume that there is $x \in A$ with $\chi(A, \mathscr{F}[X])<\chi(x, X)$. Let $\tau=$ $\chi(A, \mathscr{F}[X])$ and $\mathscr{U}$ a nbd base of $A$ in $\mathscr{F}[X]$ with $|\mathscr{U}| \leqslant \tau$. We can assume Q $=\left\{\left[A, U_{\alpha}\right]: \alpha<\tau\right\}$, where $U_{\alpha}$ is an open set of $X$ containing $A$. Since $X$ is Hausdorff, there is an open nbd $U$ of $x$ with $(A \backslash\{x\}) \cap \operatorname{cl}_{X} U=\varnothing$. Since $\chi(x, X)$ $>\tau$, there is an open nbd $V$ of $x$ with $U \cap U_{\alpha} \not \subset V$ for each $\alpha<\tau$. Let $W=V \cup$ $\left(X \backslash \mathrm{cl}_{X} U\right)$. Then $[A, W]$ is an open nbd of $A$ in $\mathscr{F}[X]$. Since $U_{\alpha} \not \subset W,\left[A, U_{\alpha}\right]$ $\not \subset[A, W]$ for each $\alpha<\tau$. Therefore, Q is not a nbd base of $A$, a contradiction. Thus, we have $\chi(x, X) \leqslant \chi(A, \widetilde{y}[X])$ for each $x \in A$. This completes the proof of Proposition 1 .

THEOREM 2. For each space $X$, the following hold:

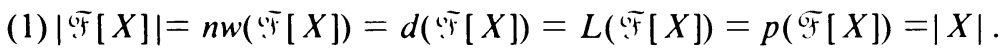

(2) $\chi(\widetilde{\mathscr{Y}}[X])=\pi \chi(\widetilde{\mathscr{Y}}[X])=\chi(X)$.

(3) $w(\mathscr{F}[X])=\pi(\widetilde{\mathscr{F}}[X])=\chi(X)|X|$.

(4) $\operatorname{psw}(\mathscr{F}[X])=\psi(\overline{\mathscr{F}}[X])=\psi(X)$.

(5) $\psi(X)=\omega$ iff $\Psi(\mathscr{F}[X])=\omega$ iff $\psi_{\Delta}(\mathscr{F}[X])=\omega$.

(6) $h d(X) h L(X) \leqslant c(\mathscr{F}[X]) \leqslant n w(X)$.

Proof. (1) Since $\{\{x\}: x \in X\}$ is a closed discrete subspace of $\mathscr{F}[X],|X| \leqslant$ $p(\mathscr{F}[X]) .|\mathscr{F}[X]|=d(\mathscr{F}[X])=|X|$ appears in [vD]. Therefore, (1) is clear.

(2) By Proposition 1, this is trivial.

(3) From

$$
\chi(X)=\pi \chi(\mathscr{F}[X]) \leqslant \pi(\mathscr{F}[X]) \quad \text { and } \quad|X|=d(\mathscr{F}[X]) \leqslant \pi(\mathscr{F}[X]),
$$


we have $\chi(X)|X| \leqslant \pi(\mathscr{F}[X]) \leqslant w(\mathscr{F}[X])$. On the other hand,

$$
w(\mathcal{F}[X]) \leqslant \chi(\mathscr{F}[X])|\mathscr{F}[X]|
$$

is always true. Therefore, by (1) and (2), (3) is proved.

(4) Since $\psi(\mathscr{F}[X]) \leqslant p s w(\mathscr{F}[X])$ is well known, we will prove $p s w(\mathscr{F}[X]) \leqslant \psi(X)$ only. For each $A \in \mathscr{F}[X]$, there is a local $\psi$-base $\mathscr{U}(A)$ of $A$ with $|\mathcal{U}(A)| \leqslant$ $\psi(\mathscr{F}[X])=\psi(X)$. Then, $\mathscr{B}=\{[A, U]: A \in \mathscr{F}[X], U \in \mathcal{Q}(A)\}$ is a pseudo base of $\mathscr{F}[X]$. Let $S \in \mathcal{F}[X] . S \in[A, U]$ iff $A \subset S \subset U$. Since $|S|<\omega,\{A: \varnothing \neq A \subset S\}$ is finite. Therefore, $\operatorname{ord}(S, \mathscr{B})=|\{[A, U] \in \mathscr{B}: S \in[A, U]\}| \leqslant \psi(X)$. Thus we have $\operatorname{ord}(\mathscr{B})=\sup \{\operatorname{ord}(S, \mathscr{B}): S \in \mathscr{F}[X]\} \leqslant \psi(X)$. Therefore, $p \operatorname{sw}(\mathscr{F}[X]) \leqslant \psi(X)$.

(5) $\psi(X)=\omega$ iff $\Psi(\mathscr{F}[X])=\omega$ appears in $[\mathbf{L}]$ and $[\mathbf{P}]$, and $\psi(\mathscr{F}[X]) \leqslant \psi_{\Delta}(\mathscr{F}[X])$ is well known. Therefore, only $\psi_{\Delta}(\mathscr{F}[X]) \leqslant \psi(\mathscr{F}[X])=\omega$ needs proof. Let $\psi(\mathscr{F}[X])$ $=\omega$ and $\mathcal{Q}(A)=\left\{U_{n}(A): n<\omega\right\}$ be a decreasing countable local $\psi$-base of each $A \in \mathscr{F}[X]$ in $\mathscr{F}[X]$. We can assume that $U_{n}(A)$ is of the form $[A, V]$, where $V$ is an open nbd of $A$ in $X$. Since $B_{n}=\cup\left\{U_{n}(A) \times U_{n}(A): A \in \mathscr{F}[X]\right\}$ is an open nbd of the diagonal $\Delta(\mathscr{F}[X])=\{(A, A): A \in \mathscr{F}[X]\}$ in $\mathscr{F}[X]^{2}=\mathscr{F}[X] \times \mathscr{F}[X], \mathscr{B}=\left\{B_{n}\right.$ : $n<\omega\}$ is a countable local $\psi$-base of $\Delta(\mathscr{F}[X])$ in $\mathscr{F}[X]^{2}$. For, let $(S, T) \in$ $\mathscr{F}[X]^{2} \backslash \Delta(\mathscr{F}[X])$. We may assume that there is an element $t \in T \backslash S$. For each nonempty $A \subset S$, there exists $n(A)<\omega$ such that $\{t\} \notin U_{n(A)}(A)$. If we put $n_{0}=$ $\max \{n(A): \varnothing \neq A \subset S\}$, then $T \notin \cup\left\{U_{n}(A): \varnothing \neq A \subset S\right\}$ for each $n \geqslant n_{0}$. Therefore, $(S, T) \notin B_{n_{0}}$ and $\mathscr{B}$ is a local $\psi$-base of $\Delta(\mathscr{F}[X])$ in $\mathscr{F}[X]^{2}$. Therefore, we have $\psi_{\Delta}(\mathscr{F}[X])=\omega$.

(6) Since $c(\mathscr{F}[X]) \leqslant n w(X)$ appears in [L], it suffices to show $h d(X) h L(X) \leqslant$ $c(\mathscr{F}[X])$.

Let us assume $h L(X)>\tau$. Then, there are a subspace $Y$ of $X$ and an open covering $\mathcal{G}$ of $Y$ such that $Y \not \subset \cup \mathcal{G}^{\prime}$ for each subfamily $\mathcal{G}^{\prime}$ of $\mathcal{G}$ with $\left|\mathcal{G}^{\prime}\right| \leqslant \tau$. We can assume that each element of $\mathcal{G}$ is open in $X$. By transfinite induction, we can easily construct $\left\{x_{\alpha}: \alpha<\tau^{+}\right\} \subset Y$ and $\left\{G_{\alpha}: \alpha<\tau^{+}\right\} \subset \mathcal{G}$ with $x_{\alpha} \in G_{\alpha} \backslash\left(\cup\left\{G_{\beta}: \beta<\alpha\right\}\right)$ for each $\alpha<\tau^{+}$. If $\beta<\alpha<\tau^{+}$, since $x_{\alpha} \notin G_{\beta}$, we have $\left[\left\{x_{\beta}\right\}, G_{\beta}\right] \cap\left[\left\{x_{\alpha}\right\}, G_{\alpha}\right]=$ $\varnothing$. Thus, $\left\{\left[\left\{x_{\alpha}\right\}, G_{\alpha}\right]: \alpha<\tau^{+}\right\}$is a pairwise disjoint family of nonempty open sets of $\mathscr{F}[X]$. Thus, we have $c(\mathscr{F}[X]) \geqslant \tau^{+}$and $h L(X) \leqslant c(\mathscr{F}[X])$.

Let $Y$ be a subspace of $X$. Consider the system $\mathscr{Q}$ of all sets of the form $[A, U]$ where $A \in \mathcal{F}[Y]$ and $U$ is an open set of $X$ containing $A$. Let $\mathscr{B}$ be a pairwise disjoint maximal family of sets in $\mathcal{Q}$. Then $D=\cup\{A:[A, U] \in \mathscr{B}\}$ is dense in $Y$. Because, assume there is $x \in Y \backslash \operatorname{cl}_{X} D$. Then, there is an open nbd $V$ of $x$ in $X$ such that $V \cap D=\varnothing$. Since $[\{x\}, V] \cap[A, U]=\varnothing$ for each $[A, U] \in \mathscr{B}, \Re \cup$ $\{[\{x\}, V]\}$ is a pairwise disjoint family of sets in $\mathcal{Q}$. This contradicts the maximality of $\mathscr{B}$. Since $|D| \leqslant|\mathscr{B}| \leqslant c(\mathscr{F}[X])$, we have $d(Y) \leqslant c(\mathscr{F}[X])$. Thus, $h d(X) \leqslant c(\mathscr{F}[X])$ is proved. This completes the proof of Theorem 2 .

Hajnal and Juhász studied conditions when $\mathscr{F}[X]$ has countable cellularity. Readers, who take interest in this case, refer to [HJ].

Since $\mathscr{F}[X]$ is regular [vD], we have the following:

COROLlaRY 3. In the following, (1)-(5) are equivalent, and each of them implies (6).

(1) $X$ is countable.

(2) $\mathscr{F}[X]$ is countable. 
(3) $\mathscr{F}[X]$ is a cosmic space.

(4) $\mathscr{F}[X]$ is (hereditarily) separable.

(5) $\mathscr{F}[X]$ is (hereditarily) Lindelöf.

(6) $\mathscr{F}[X]$ is paracompact.

LeMma 4. If $X$ is a paracompact Hausdorff space, then $L(X) \leqslant c(X)$.

Proof. Since each open covering of $X$ has a $\sigma$-discrete open refinement, the lemma is clear.

COROllary 5. If $n w(X)<|X|$ holds, then $\mathscr{F}[X]$ is not paracompact. In particular, if $X$ is an uncountable separable metric space, then $\mathcal{F}[X]$ is not metrizable.

The following corollaries are also clear.

COROllaRY 6. If $\mathscr{F}[X]$ has $G_{\delta}$-diagonal, then the pseudo character of $X$ is $\omega$.

COROLlaRY 7. If $\mathscr{F}[X]$ has countable cellularity, then $X$ is both hereditarily separable and hereditarily Lindelöf.

THEOREM 8. Let $\phi$ be a cardinal function listed above except $p s w, \psi_{\Delta}$ and $\Psi$. Then, we have $\phi(X) \leqslant \phi(\mathscr{F}[X])$ for each space $X$.

Proof. $t(X) \leqslant t(\mathscr{F}[X])$ follows from Proposition 1. Proofs for other cardinal functions follow from Theorem 2 .

ExAmple 1. By (4) in Theorem 2, we have always $p s w(\mathscr{F}[X]) \leqslant p s w(X)$. There is a compact Hausdorff GO-space $X$ for which $p s w(\mathscr{F}[X])<p s w(X), \psi_{\Delta}(\mathscr{F}[X])<\psi_{\Delta}(X)$ and $\Psi(\mathcal{F}[X])<\Psi(X)$ hold. For example, let $X$ be the lexicographic ordered square [E, 3.12.3]. Then $p s w(X)=\psi_{\Delta}(X)=\Psi(X)=c>\omega=\psi(X)$. Therefore, we have $\operatorname{psw}(\mathscr{F}[X])=\psi_{\Delta}(\mathscr{F}[X])=\Psi(\mathscr{F}[X])=\omega$ by Theorem 2 .

EXAMPLE 2. There is a compact Hausdorff GO-space $X$ for which $h d(X) h L(X)<$ $c(\mathscr{F}[X])=n w(X)$ holds. In fact, let $X$ be the two arrows space [E, 3.10.C]. That is, $X=\{(x, 0): 0<x \leqslant 1\} \cup\{(x, 1): 0 \leqslant x<1\}$ considered as a subspace of the lexicographic ordered square. Then, $h d(X)=h L(X)=\omega<c=n w(X)$. Let us take a point $P(x)=(x, 0)$ in $X$. Since $U(x)=\{(x, 0),(0,1)\} \cup\{(y, i): 0<y<x$, $i=0,1\}$ is an open nbd of $P(x),\{[\{P(x)\}, U(x)]: 0<x \leqslant 1\}$ is a disjoint family of open sets of $\mathscr{F}[X]$. Therefore, we have $c(\mathscr{F}[X])=c$.

Example 3. There is a $T_{1}$-space $X$ which has a finite subset $A$ such that $\chi(A, \mathscr{F}[X])<\max \{\chi(x, X): x \in A\}$ holds. Let $X$ be the set of all lattice points $(i, j)$ of positive integers with two ideal points $p$ and $q$. The topology of $X$ is defined by declaring each lattice point to be open, and by taking as open nbds of $p$ sets of the form $X \backslash(B \cup\{q\})$ where $B$ is any set of lattice points with at most finitely many points on each row, and as open nbds of $q$ sets of the form $X \backslash(C \cup\{p\})$ where $C$ is any set of lattice points selected from at most finitely many rows. Then $X$ is a compact $T_{1}$-space and $\chi(p, X)>\omega$ holds [SS, 99]. Let $A=\{p, q\}$. If $U$ is a nbd of $A$, then $|X \backslash U|<\omega$. Therefore, $\mathscr{B}=\{[A, U]: A \subset U \subset X,|X \backslash U|<\omega\}$ is a nbd base of $A$ in $\mathscr{F}[X]$. Since $|\mathscr{B}|=\omega$, we have $\chi(A, \mathscr{F}[X])<\chi(p, X)$.

Concerning theorems and examples, we ask the following.

Question 1. Let $\phi \in\left\{\Psi, \psi_{\Delta}\right\}$. Does $\phi(\mathscr{F}[X]) \leqslant \phi(X)$ hold for each space $X$ ? 
Question 2. Determine exactly $t, \Psi$ and $\psi_{\Delta}$ on $\mathscr{F}[X]$ in terms of those on $X$. Lutzer asked this question for the cellularity $c[\mathbf{L}]$.

\section{REFERENCES}

[vD] E. K. van Douwen, The Pixley-Roy topologv on spaces of subsets, Set-theoretic Topology, Academic Press, New York, 1977, pp. 111-134.

[E] R. Engelking, General topologv, PWN, Warsaw, 1977.

[HJ] A. Hajnal and I. Juhász, When is a Pixley-Roy hyperspace CCC?. Topology Appl. 13 (1982), 33-41.

[J] I. Juhász, Cardinal functions in topology - ten years later, Math. Centre Tracts, No. 123, North-Holland, Amsterdam, 1980.

[L] D. J. Lutzer, Pixley-Roy topology, Topology Proc. 3 (1978), 139-158.

[P] T. C. Przymusinski, Normality and paracompactness of Pixley-Roy hyperspaces, Fund. Math. 113 (1981), 201-219.

[SS] L. A. Steen and J. A. Seebach, Jr., Counterexamples in topology, Springer-Verlag, Berlin and New York, 1978.

Faculty of Liberal Arts, Shizuoka University, Ohya, Shizuoka, 422, Japan 